\title{
RESUSPENSION OF PLATELETS:
}

\section{ENHANCED 5-HYDROXYTRYPTAMINE-INDUCED AGGREGATION IN CHLORPROMAZINE TREATED PATIENTS DUE TO CHANGES IN PLATELET PROPERTIES}

\author{
D.J. BOULLIN, D.G. GRAHAME-SMITH, R.P.J. GRIMES \& H.F. WOODS \\ MRC Unit and Department of Clinical Pharmacology, Radcliffe Infirmary, Woodstock Road, Oxford OX2 6HE
}

1 Previous work showed that platelets from patients receiving chlorpromazine (CPZ) have an enhanced aggregation response to 5-HT. This was caused by an action of CPZ on the platelets, a factor borne by the plasma, or both.

2 Therefore we studied 5-HT induced aggregation of platelets from CPZ treated patients resuspended in normal plasma and from normal subjects resuspended in CPZ plasma.

3 Aggregation responses of normal platelets to 5-HT were not enhanced by resuspension either in normal plasma or in CPZ plasma. In addition the enhanced responses of platelets from $\mathrm{CPZ}$ treated patients did not revert to normal after resuspension in normal plasma.

4 It is concluded that the enhanced aggregation response to 5-HT seen after CPZ treatment is due to a change in the properties of the platelets rather than a factor in the plasma.

\section{Introduction}

In the previous paper we showed that patients receiving chlorpromazine (CPZ) have enhanced platelet aggregation in vitro in response to low concentrations of 5-hydroxytryptamine (5-HT) (Boullin, Gelder, Grahame-Smith, Grimes, Kolakowska, Wiles \& Woods, 1975). These responses have also been reproduced in animals (Boullin, Bromley, Grahame-Smith, Grimes \& Starling, unpublished observations).

The question arose as to whether the effect produced by $\mathrm{CPZ}$ treatment in man resulted from an action of the phenothiazine on the platelets, to a factor circulating in the plasma or both. Accordingly, we have studied the effect of 5-HT upon platelets from CPZ treated patients resuspended in normal plasma and the responses of normal platelets resuspended in plasma from $\mathrm{CPZ}$ treated patients.

The results indicate that there is no factor circulating in the plasma at the time our experiments were made which is responsible for the change in 5-HT induced aggregation, but the properties of the platelet itself are altered.

\section{Methods}

The procedure for obtaining platelet rich plasma (PRP) and for investigating aggregation have been described in our earlier papers (Boullin et al.,
1975; Boullin, Grahame-Smith, Grimes \& Woods, 1974).

The sources of the patients and control were also described previously (Boullin et al., 1974).

The subjects were nine CPZ treated patients, of either sex (patients); the controls were three normal volunteers from our laboratory plus two patients who were not receiving phenothiazine therapy (control).

\section{Separation and resuspension of platelets}

Platelets were separated from their plasma and resuspended in fresh plasma by the following modification of the method of Ganguly \& Sonnichsen (1973): Citrated PRP (5 ml) (Boullin et al., 1974) was layered onto sodium metrizoate (1 $\mathrm{ml})$ (Hypaque, Winthrop Laboratories, Surbiton-upon-Thames, Surrey) which had been diluted with distilled water to give a specific gravity of 1.1. After layering in a $7 \mathrm{ml}$ polycarbonate centrifuge tube the PRP: metrizoate interface was gently disturbed in order to produce a density gradient. The solutions were then centrifuged at $1200 \mathrm{~g}$ for $15 \mathrm{~min}$ in an MSE Mistral $6 \mathrm{~L}$ centrifuge in a swinging bucket rotor at $22^{\circ} \mathrm{C}$. The platelets sedimented in a band at the plasma: metrizoate interface while the heavier erythrocytes and leucocytes passed to the bottom of the tube (Ganguly \& Sonnichsen, 1973). The 


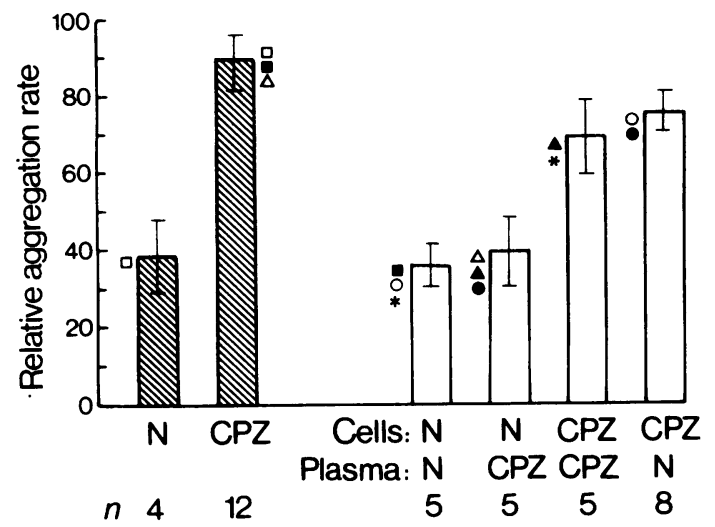

Figure 1 Effects of resuspension of platelets from control (N) and chlorpromazine treated (CPZ) subjects on platelet aggregation responses to 5-HT. The results are expressed in terms of relative aggregation rate

\section{(5-HT aggregation rate $\times 100)$}

during $15 \mathrm{~s}$ after addition of 5-HT to PRP. Aggregation was investigated in autologous plasma prior to resuspension (hatched columns) or after resuspension (open columns) under the conditions shown on the abscissa. Values are the mean \pm s.e. mean obtained with the number of subjects indicated (n). Significant differences between the various groups are shown by: $\triangle P<0.05$; $\square \Delta P<0.01$; - * $\circ$ $P<0.001$.

platelets were removed and resuspended in sufficient plasma to restore the platelet count $/ \mathrm{ml}$ plasma to the original value, as measured with the Coulter Counter (Boullin et al., 1974).

As sodium metrizoate was found to inhibit 5-HT induced aggregation, it was removed by dialysis against $200 \mathrm{vol}$ of calcium-free Tyrodes solution (Boullin, 1967) for $2.5 \mathrm{~h}$ at $22^{\circ} \mathrm{C}$. This dialysis was found to restore the aggregation responses to 5-HT to normal.

Resuspension of the isolated platelets after dialysis was made according to the following schedule:

Control platelets in control plasma $(\mathrm{N}+\mathrm{N})$

Control platelets in patients' plasma $(\mathrm{N}+\mathrm{CPZ})$

Patients' platelets in patients' plasma $(\mathrm{CPZ}+\mathrm{CPZ})$

Patients' platelets in control plasma $(\mathrm{CPZ}+\mathrm{N})$

Aggregation responses to $20 \mathrm{nmol} / \mathrm{ml} 5-\mathrm{HT}$ or ADP were quantitated as changes in initial aggregation rate and the total change in optical density in $210 \mathrm{~s}$ as described earlier (Boullin et al., 1974).

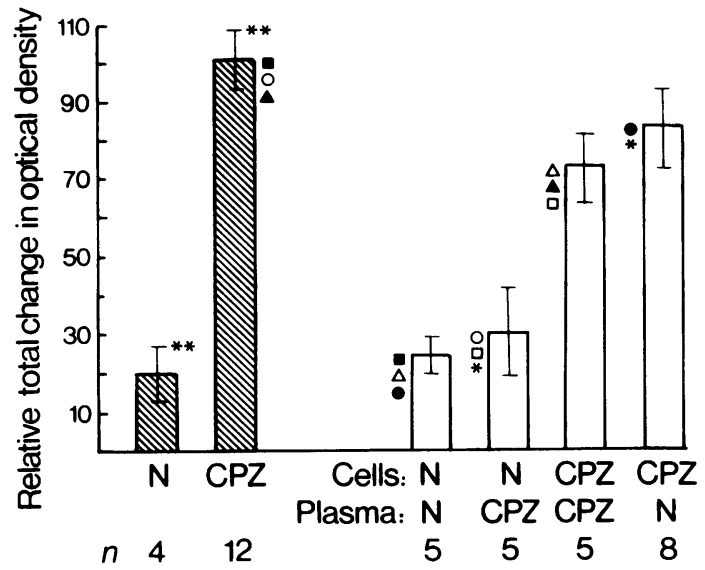

Figure 2 Effect of resuspension of platelets from control subjects (normal) and CPZ treated patients (CPZ) on platelet aggregation responses to $5-\mathrm{HT}$. The results are expressed as the total change in optical density produced by 5-HT in $210 \mathrm{~s}$ in terms of the maximum change produced by ADP. Aggregation was investigated in autologous plasma prior to resuspension (hatched columns) or after resuspension (open columns) under the conditions shown on the abscissa. Values are the mean \pm s.e. mean obtained with the number of subjects indicated $(n)$. Significant differences between the various groups are shown by: $\triangle P<0.05 ; \triangle, \circ,{ }^{*} P<0.01 ; \bullet,{ }^{*} *, 0, \bullet P<0.001$.

Results

There were wide individual variations in aggregation and responses as reported earlier Boullin et al. (1974). To allow for this we have followed our earlier procedure of expressing the changes in rate and optical density produced by 5-HT in terms of the maximum response produced by ADP.

\section{Aggregation responses prior to resuspension}

We confirmed our earlier results that the responses of patients to 5-HT were enhanced. In 12 patients the 5-HT aggregation rate was $89.4 \pm 7.7 \%$ of the ADP rate whereas in 4 controls the rate was $38.3 \pm 9.3 \%$ of the ADP rate.

All subsequent responses were compared in terms of aggregation rate (Figure 1) and change in optical density (Figure 2) with those described here.

\section{Aggregation responses after resuspension}

Control responses. Aggregation responses of control platelets to 5-HT were not changed by 
resuspension. This was the case whether the cells were replaced in control plasma $(\mathrm{N}+\mathrm{N})$ or in plasma from patients $(\mathrm{N}+\mathrm{CPZ})$.

There was no significant changes in rate (Figure 1 ) or optical density (Figure 2).

Responses of platelets from CPZ treated patients. When patients' platelets were resuspended in patients' plasma $(\mathrm{CPZ}+\mathrm{CPZ})$ there was no significant reduction in aggregation rate or change in optical density in comparison with responses prior to resuspension. Following resuspension in control plasma the enhanced 5-HT responses persisted (Figures 1 and 2).

\section{Discussion}

The main facts which have come out of this work are: first, platelets from CPZ treated patients still show the enhanced aggregation response when placed in normal plasma; second, normal platelet responses remained unchanged when the cells are resuspended in plasma from $\mathrm{CPZ}$ treated patients.

We conclude that CPZ therapy in some way alters the properties of the platelet so that the aggregation response to $5-\mathrm{HT}$ is enhanced. There are at least two possibilities, either a long-term direct effect of $\mathrm{CPZ}$ or metabolites on the platelet itself or that $\mathrm{CPZ}$ treatment results in the synthesis and/or release of some unidentified substance which acts on the platelet to produce the response described. This compound could be synthesized either within the platelet itself or at some remote site and carried to the platelet via the circulation.

A direct phenothiazine effect could involve a change in 5-HT transport across the platelet

\section{References}

BAUMGARTNER, H.R. (1969). 5-hydroxytryptamine uptake and release in relation to aggregation of rabbit platelets. J. Physiol. (Lond.), 201, 409-423.

BAUMGARTNER, H.R. \& BORN, G.V.R. (1969). The relation between the 5-hydroxytryptamine content and aggregation of rabbit platelets. J. Physiol. (Lond.), 201, 397-408.

BOULliN, D.J. (1967). The action of Extracellular cations on the release of the sympathetic transmitter from peripheral nerves. J. Physiol., 189, 85-99.

BOULLIN, D.J., GRAHAME-SMITH, D.G., GRIMES, R.P.J. \& WOODS, H.F. (1974). Inhibition of human membrane. Baumgartner (1969) and Baumgartner \& Born (1969) proposed that 5-HT induced aggregation of rabbit platelets required the net transport of the indoylethylamine across the plasma membrane of the cell. Inhibition of aggregation was associated with saturation of the energy-dependent transport process. It is not known whether this theory can be applied to 5-HT induced aggregation of human platelets, but if so then the enhancement of aggregation seen in CPZ treated patients would require more rapid net transport of 5-HT. Preliminary experiments do not show any evidence of an increased rate of transport of 5-HT in these patients (Boullin, Grahame-Smith, Grimes \& Woods, unpublished observations).

Regarding the synthesis or release of an unidentified substance we have no information at present other than the fact that it does not appear to be in the plasma at the time our experiments were made. However, there is some supporting evidence for an indirect action from evidence obtained with human platelets in vitro and animal platelets in vivo. Our earlier in vitro experiments (Boullin et al., 1974) showed that CPZ and seven metabolites all inhibited 5-HT induced aggregation. Moreover in unpublished experiments with goats (Boullin, Bromley, Grahame-Smith, Grimes \& Starling), we find that i.v. CPZ $(6 \mathrm{mg} / \mathrm{kg})$ inhibits 5-HT induced aggregation $30 \mathrm{~min}$ after injection but produces a pattern of enhanced responses after $4 \mathrm{~h}$ typical of these seen in $\mathrm{CPZ}$ treated humans. Thus the established inhibition of platelet aggregation produced when CPZ is added to human platelet rich plasma in vitro can be reproduced in vivo in animals before the appearance of enhanced responses very similar to those seen in man. blood platelet aggregation by chlorpromazine and its metabolites. Br. J. Pharmac., in press.

BOULLIN, D.J. GELDER, M., GRAHAME-SMITH, D.G., GRIMES, R.P.J., KOLAKOWSKA, T., WILES, D. \& WOODS, H.F. (1975). Increased platelet aggregation responses to 5-hydroxytryptamine in patients taking chlorpromazine. Br. J. clin. Pharmac., 2, 29-35.

GANGULY, P. \& SONNICHSEN, W.J. (1973). A simple method for the isolation of blood platelets. J. clin. Path., 26, 635-637.

(Received October 10, 1974) 